\title{
A STUDY ON 2-D VECTOR FLOW MAPPING BY ECHO-PIV WITH TOTAL FOCUSING METHOD
}

\author{
TAKUYA KAWACHI ${ }^{1}$, HIDEHARU TAKAHASHI ${ }^{2} \&$ HIROSHIGE KIKURA $^{2}$ \\ ${ }^{1}$ Tokyo Institute of Technology, Japan \\ ${ }^{2}$ Laboratory for Advanced Nuclear Energy, Tokyo Institute of Technology, Japan
}

\begin{abstract}
Optical techniques have been applied for inspecting inside the primary containment vessels (PCV) in the decommissioning of the Fukushima Dai-ichi nuclear power plant. In this inspection, the following are required: determining fuel debris distribution and identifying location of leakage. Until this data, one of the inspections achieved great progress by capturing several images of fuel debris using a video camera but no information of the leak was unveiled due to non-clear water causing poor visibility of that camera. In order to identify the location of the leak, a new imaging method combining diverging wave (DW) and total focusing method (TFM), named DW-TFM was proposed aimed at echo (ultrasound)-particle image velocimetry (PIV) since an ultrasound can be used in opaque liquid and ultrasonic transducers are generally suited to high radiation levels. This imaging method uses an ultrasonic array sensor and emits a DW spreading in the distance from that sensor. The echo signal of tracer particles where the DW passed is captured by all the elements in the sensor at the same time and an echo image is then reconstructed from those signals using TFM algorithm. The imaging method is expected to obtain echo images covering a wide range in one transmission. Echo-PIV uses consecutive echo images captured at a certain interval to process them with PIV algorithm and obtain an averaged vector flow map (VFM). Summarizing the above, echo-PIV using the DW-TFM has the potential to visualize a wide range of flow behavior in real time and it allows the location of the leak to be found quickly. After investigating the imaging performance of the DW-PIV and developing a measurement system for the suggested echo-PIV, experimental measurement was conducted under a simple condition of water leaking from a tank in order to confirm its applicability. In this test, leaking position could be assumed from the measured VFM and it has good agreement with the actual leakage position. The achievability of the method for the leakage point detection is hereby presented.

Keywords: ultrasound, total focusing method, diverging wave, echo-PIV, vector flow mapping, identification of leakage, decommissioning of Fukushima Dai-ichi NPP.
\end{abstract}

\section{INTRODUCTION}

As a part of the decommissioning of the Fukushima Dai-ichi nuclear power plant (1F), an inspection of the fuel debris has recently been conducted inside the primary containment vessel (PCV) of units 1-3. For an efficient fuel debris removal, the location of the fuel debris is required. Moreover, detecting the location of leakages is necessary to stop the leaking of contaminated water. Until this date, several inspections have been performed using some robots with video camera and one of those conducted on July 2017 achieved a significant progress by capturing the first images of substance regarded as a fuel debris (Fig. 1) [1]. However, the inspection has not yet found any locations of leakage, plus it unveiled that an optical inspection is not suitable because the retained water is not clear. Therefore, other inspection methods are required to accelerate the progress of the inspection inside the PCVs.

In this work, an ultrasonic measurement technique is considered as a promising nonoptical inspection method because an ultrasound can be used in opaque liquids and overcome problems with optical techniques. Also, ultrasonic transducers are generally suited to high radiation levels since it has been used in the decommissioning of the Three Mile Island [2] and even applied to treat radioactive wastewater [3]. That means the ultrasonic measurement techniques can be suitable for the state inside the PCV. 

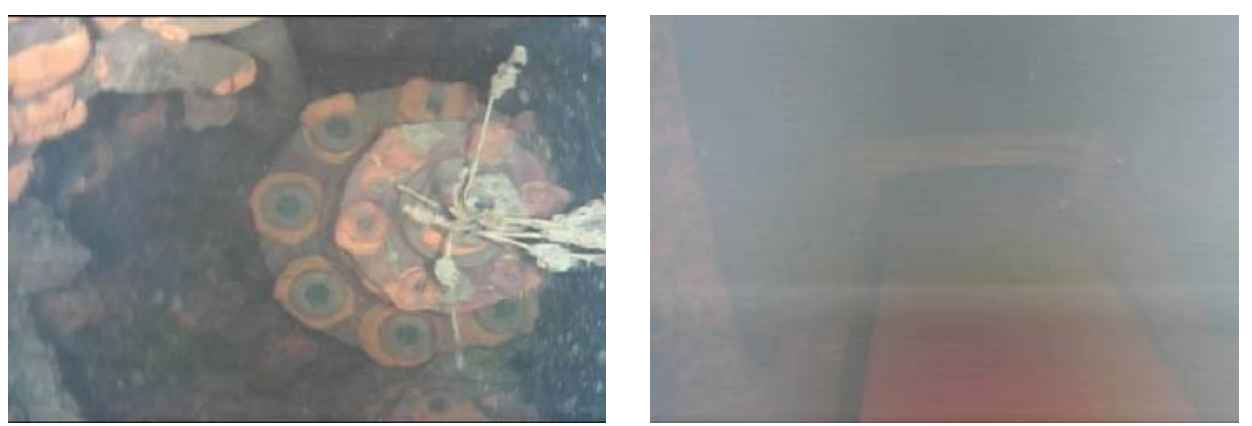

Figure 1: Images captured inside the PCV at unit 3 [1].

Ultrasonic velocity profiler (UVP) is a typical candidate for measuring the flow behavior of the water and thus effectively detecting the leakages. Using a single transducer, this method can obtain a velocity profile with only one component along the measurement line. Measuring an actual vector of velocity requires at least two measurements from different angles. Therefore, coupled sensors such as using two convex array sensors [4] are used for obtaining vector flow map (VFM). However, in the case of the inspection, such coupled sensors do not seem to be suitable since the whole system becomes large (especially for a large inspection area) and the current access to the PCVs is limited. A phase array UVP [5] with a compact sensor is preferable for the inspection. In the phase array UVP, an array senor is used and the emitted beam is steered by a corresponding delay between the excitation of each element in that array. Two elements capture the echo signal of tracer particles in the steered beam from different angles. The profile with two components of the velocity vector can be estimated using two velocity profiles calculated with UVP from both echo signals. VFM can be then obtained by changing the steering angle. This method allows the measurement in a wide range using a small array sensor. However, the phased array UVP requires a long scanning time for obtaining a good spatial resolution of the VFM even though a quick measurement is needed for a high inspection efficiency.

Our present works focused on the echo-PIV method for its high potential of a much faster acquiring of the VFM. In this method, several echo images are captured at a certain time interval using an array sensor and VFM can be estimated by video-processing with PIV (Particle Image Velocimetry) algorithm afterwards. Echo-PIV proposed in [6] applies sector scan using a convex array sensor to capture the echo image. This imaging method can obtain a wide-range echo image, which is PIV-grade only in the area near the senor since azimuthal resolution becomes much worse farther from the sensor in proportion to the curvature of the convex array sensor. Thus, the imaging method is not preferable for the inspection. The total focusing method (TFM) [7] is an advanced imaging method well-known in the ultrasonic non-destructive testing field. In this method, a linear array senor is used and the echo signal is received by all elements after exciting one element in that sensor. Echo image is reconstructed from the received signals and it has a high azimuth resolution even far from the sensor. The classical TFM called the full matrix capture (FMC) captures echo signals from all combinations of transmitter-receiver pairs in the array sensor by an acquisition scheme of one transmitting element and all receiving elements and reconstructs the echo image using that data to achieve the best resolution and signal-noise ratio (SNR) in wide range. However, this method is not suited to echo-PIV because the target tracer is moving during the acquisition of the FMC data and thus it may cause an error in reconstructing the 
echo image. Also, the classical TFM cannot achieve high time resolution of echo images. In order to avoid this problem, the diverging wave (DW) [8] was applied as a transmission scheme. This DW is emitted by elements excited with time delays as if a virtual point source was deployed in the rear of the array transducer and can cover wide range with relatively high SNR only in one transmission. With this in mind, a new imaging method combined of DW and TFM, named DW-TFM was proposed. This DW-TFM is expected to obtain PIVgrade echo image in wide range, dealing with the problem of the classical TFM on carrying out echo-PIV.

The target of this study is to develop the measurement system for echo-PIV using the DWTFM. This paper describes the following steps of the development: the investigation of the imaging performance of the DW-TFM, the development of the echo-PIV measurement system and the verification experiment and results.

\section{INVESTIGATION OF THE IMAGING PERFORMANCE}

In this section the DW-TFM is formulized at first. Subsequently, the acoustic field when transmitting DWs is simulated to find an optimal virtual source maximizing the measurable area in one transmission. Eventually, the imaging performance of the DW-TFM is experimentally investigated.

\subsection{Formulization of the DW-TFM}

The DW-TFM uses a linear array sensor. A virtual point source is deployed in the rear of the array sensor as shown in Fig. 2. An ultrasonic pulse is emitted from each of the elements with time delays corresponding to the emission from the virtual source. The time delay applied at each element is expressed as:

$$
\Delta \tau_{t e}=\frac{\left(\sqrt{\left(x_{t e}-x_{v}\right)^{2}+z_{v}^{2}}-\min \left(\sqrt{\left(x_{t e}-x_{v}\right)^{2}+z_{v}^{2}}\right)\right)}{c},
$$

where $x$ and $z$ are coordinates of the element position, subscript of te and $v$ are the index of transmitting element and the virtual source, respectively, $\Delta \tau$ is the time delay applied and $c$ is sound speed of the medium.

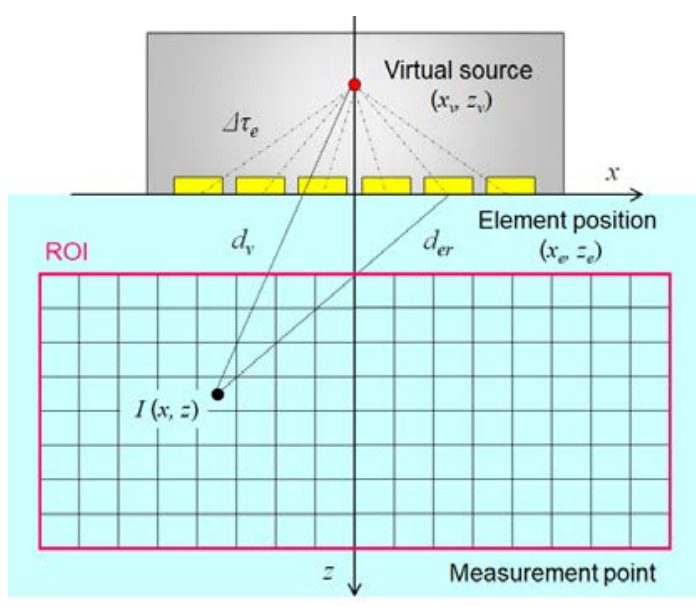

Figure 2: Definition of parameters for DW-TFM. 
The diverging wave called DW is the wavefront which is formed by all the transmitted pulses. After the transmitting, the echo signal is received by all the elements in the transducer, and an echo image is reconstructed from the received signals in the post-processing of the TFM. The imaging equation is expressed as:

$$
I(x, z)=\left|\sum_{r e=1}^{N_{e}} \frac{H_{r e}\left(\frac{d_{v}}{c}+\frac{d_{r e}}{c}\right)}{\sqrt{d_{v} d_{r e}}}\right|,
$$

where subscript of re is the index of the receiving element, $I$ is the echo intensity, $N_{e}$ is the number of elements, $H$ is a Hilbert transform of the received echo signal and $d$ is the distance between two positions.

\subsection{Acoustic field simulation}

Several methods have been suggested to simulate the acoustic filed. In this study, the distributed point source method (DPSM) [9] was adopted since our presented research [10] has validated it by comparing it with measured data.

A small laboratory scale sensor was used. Its specification was the following: the center frequency was $4 \mathrm{MHz}$, the number of elements was 16 , the element width $a$ was $0.15 \mathrm{~mm}$, the element length $b$ was $3.0 \mathrm{~mm}$ and the pitch between two adjacent elements was $0.2 \mathrm{~mm}$.

Fig. 3 shows the frequency characteristic of the array sensor. This frequency characteristic was experimentally investigated along the following steps; a $90 \mathrm{~mm}$ cubic acrylic block was placed perpendicularly to the array sensor. The both were immersed in water. A pulse of 5 cycles was emitted from one of the elements and echo signal from the interface was analysed afterwards. The frequency characteristic is required for simulating the acoustic field.

For simulating the acoustic field, two types of mesh were used. The first mesh was set as $-75 \leq x \leq 75 \mathrm{~mm}, 0 \leq z \leq 150 \mathrm{~mm}$ on $x-z$ plane $(y=0 \mathrm{~mm})$. The second mesh was for $-75 \leq x \leq 75 \mathrm{~mm},-20 \leq y \leq 20 \mathrm{~mm}, z=25,75$ or $125 \mathrm{~mm}$. Both mesh types have used the mesh vertex distance of $0.5 \mathrm{~mm}$ and the coordinates shown in Fig. 4. The time step was $T / 10$ with $T$ being the period of the center frequency. Virtual source was deployed at $x=y=0$ and $Z$ varying from -20 to $0 \mathrm{~mm}$ (step size: $0.1 \mathrm{~mm}$ ). The acoustic field was calculated for each of these source positions.

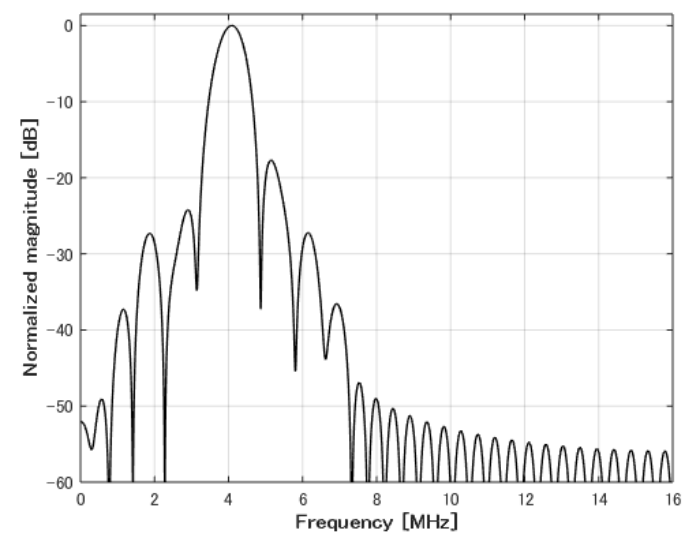

Figure 3: Frequency characteristic of the array sensor. 


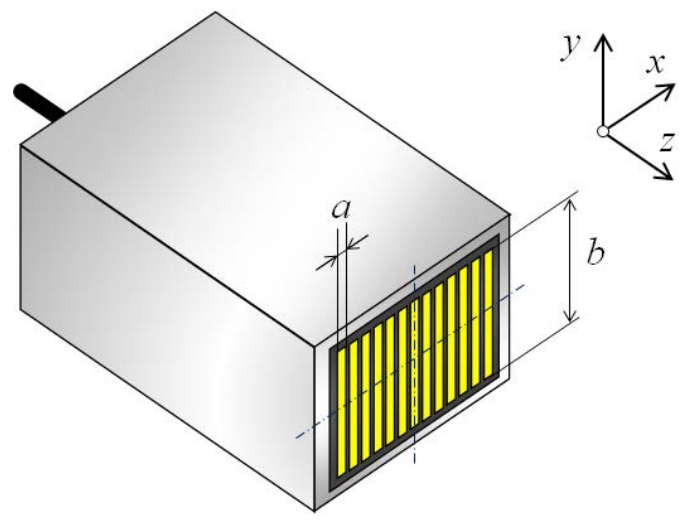

Figure 4: Definition of 3-D coordination and configuration of the array sensor.

Figs 5 and 6 show the acoustic field calculated when the virtual source was located at $z=-3.1 \mathrm{~mm}$. The maximum amplitude in the elapsed time was extracted at each point of the mesh and color-mapped in decibels. As shown in Fig. 5, the result indicated the widest measurable area on the $x-y$ plane as compared with the other virtual sources in the range over $-40 \mathrm{~dB}$. The beam shape shows the diverging wave spreads within the two broken lines denoting $\pm 30^{\circ}$ angles. Although for $z=50-80 \mathrm{~mm}$, the amplitude on the broken lines was weak less than $-40 \mathrm{~dB}$, the virtual source allows to measure the area roughly up to $150 \mathrm{~mm}$ far within $\pm 30^{\circ}$. This area is defined as the measurement area. Fig. 6 shows the cross section of the beam at $z=25,75$ and $125 \mathrm{~mm}$ from the top to the bottom, respectively. The results indicate that the thickness of the beam increases with the distance from the sensor and that side lobes appear. This lowers the resolution in the $y$ direction. However, the effect of the side lobes might be ignored because the amplitude of them is sufficiently lower than that of the main lobe.

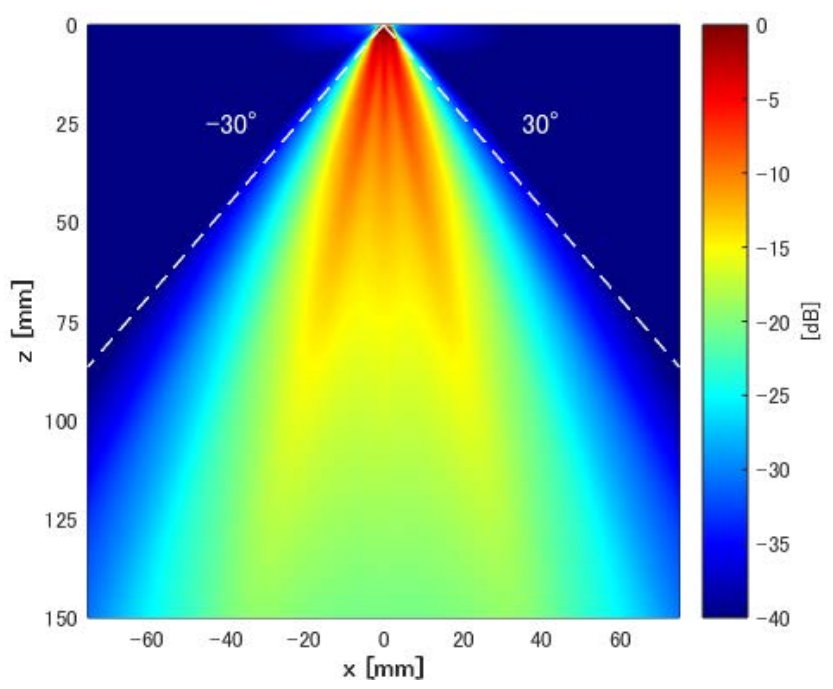

Figure 5: Simulated beam shape on $\mathrm{x}-\mathrm{z}$ plane. 


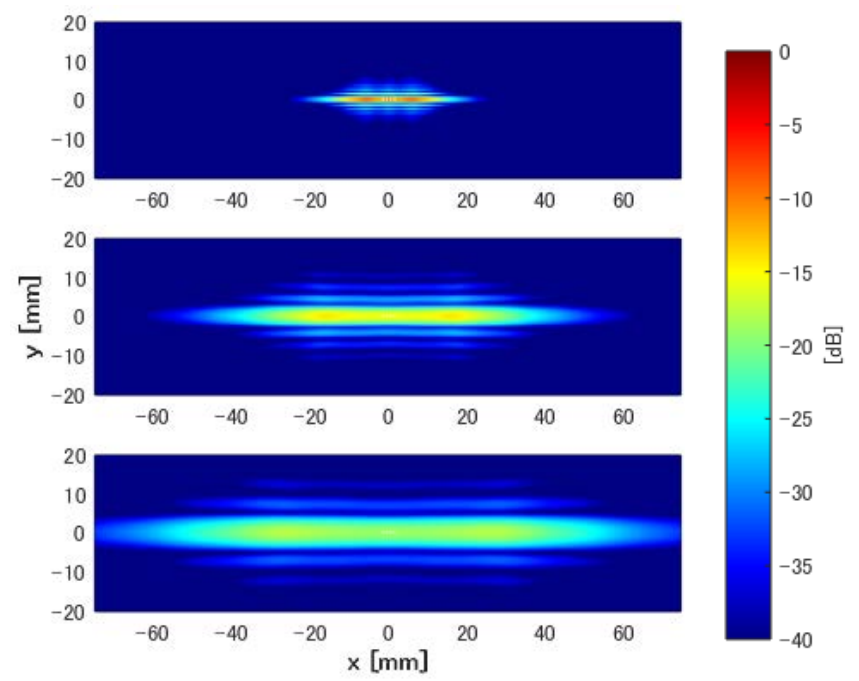

Figure 6: Simulated beam cross-section shape for $z=25,75$ and $125 \mathrm{~mm}$ (top, center and bottom respectively).

In consequence, the optimal virtual source position was determined to be at $z=-3.1 \mathrm{~mm}$ and the corresponding beam allows to measure in the range up to $150 \mathrm{~mm}$ far and within $\pm 30^{\circ}$ but the low resolution in the $y$ direction should be only concerned.

\subsection{Investigation of the imaging performance}

Experiment was conducted to evaluate the imaging performance of the DW with the optimal virtual source. The measurement device consisted of a linear array sensor, a pulser/receiver (JAS21 model 32CH, Japan Probe Co. Ltd.) and a control PC. Fig. 7 shows the schematic of the experimental apparatus used in this experiment. Eleven wires $(\varphi 0.28 \mathrm{~mm})$ fixed to two

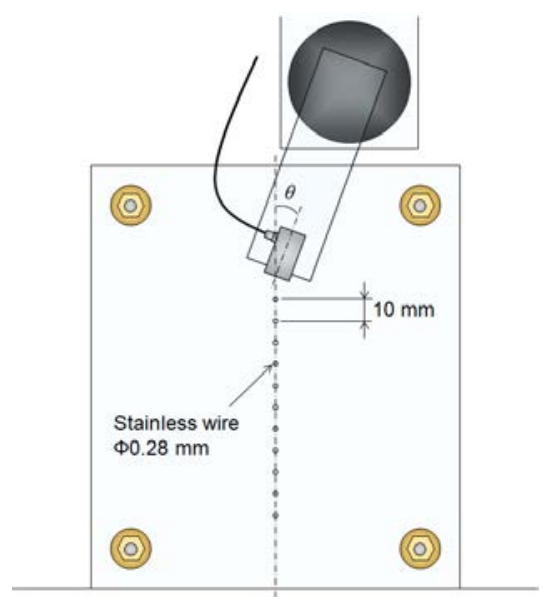

Figure 7: Schematic experimental apparatus. 
acrylic plates at $10 \mathrm{~mm}$ intervals were straightened using a slight tension and used as scatterers instead of tracer particles. The array sensor was set $10 \mathrm{~mm}$ above the first wire. The data acquisition was carried out for the sensor's angle $\theta$ from $0^{\circ}$ to $30^{\circ}$ with $5^{\circ}$ steps. The condition of the data acquisition was as follows: the applied voltage was $150 \mathrm{~V}$, each pulse had 5 cycles, the returning signal was filtered using a high-pass filter $(1 \mathrm{MHz})$ and a low-pass filter ( $7 \mathrm{MHz}$ ), the sampling rate was $32.5 \mathrm{MHz}$ and the gain was $25 \mathrm{~dB}$.

Fig. 8 shows the imaging result of DW-TFM at $\theta=20^{\circ}$ as an example. To determine the azimuthal resolution, an one-dimensional echo intensity distribution was extracted on each radius from the array sensor corresponding to the wire positions (see the pink broken line drawn in the enlarged image). Each of them was normalized with its maximum value and the full width half maximum (FWHM) was defined as azimuth resolution at the position.

Fig. 9 shows the relationship between the HWHM, the measuring distance and the angle of the DW-TFM. This result indicates that the HWHM tends to increase in proportion to the distance (radius) and does not depend on the measuring angle except in the case of $30^{\circ}$. The reason for the change at $30^{\circ}$ is the weak amplitude for this angle farther than $60 \mathrm{~mm}$ from the sensor as mentioned in Section 2.2 (see Fig. 5). The HWHMs are less than $20 \mathrm{~mm}$ in the over-all length. Fig. 10 shows the difference between the HWHM with the DW-TFM and with the classical TFM as expressed by $\left(H_{d}-H_{c}\right) / H_{c} \times 100$. Where $H_{d}$ and $H_{c}$ are HWHM of the DW and the classical TFM, respectively. This result indicates that the difference remains relatively even except for the positions farther than $60 \mathrm{~mm}$ at $30^{\circ}$ and this implies that the classical TFM has the same tendency regarding the relationship between HWHM, the measuring length and the angle. The HWHM of the DW was approximately $35 \%$ larger than that of the classical TFM, except for the positions farther than $60 \mathrm{~mm}$ at $30^{\circ}$ but is not much worse despite of the fact that the transmission of the classical TFM (equals use of the data amount for the imaging) is 16 times bigger than that of the DW-TFM.

In conclusion of the investigation, the image quality of DW-TFM is not much worse than the classical TFM and should be sufficient for the PIV processing.

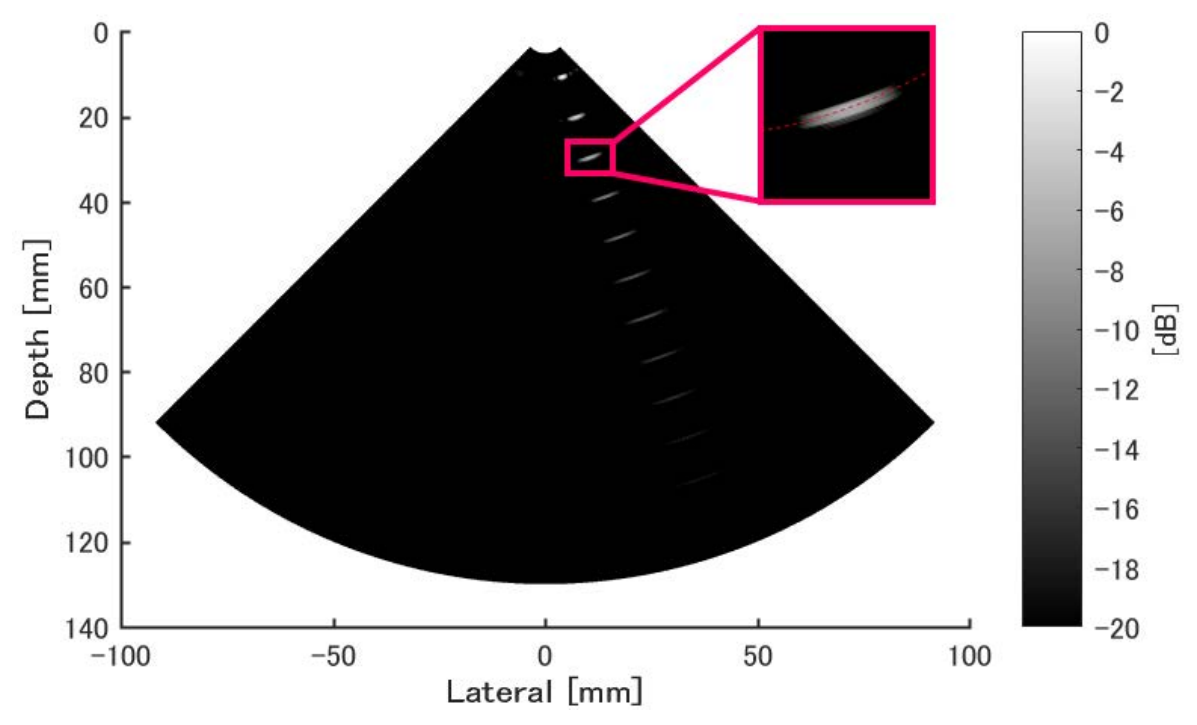

Figure 8: Imaging result with DW-TFM at $\theta=+20^{\circ}$. 


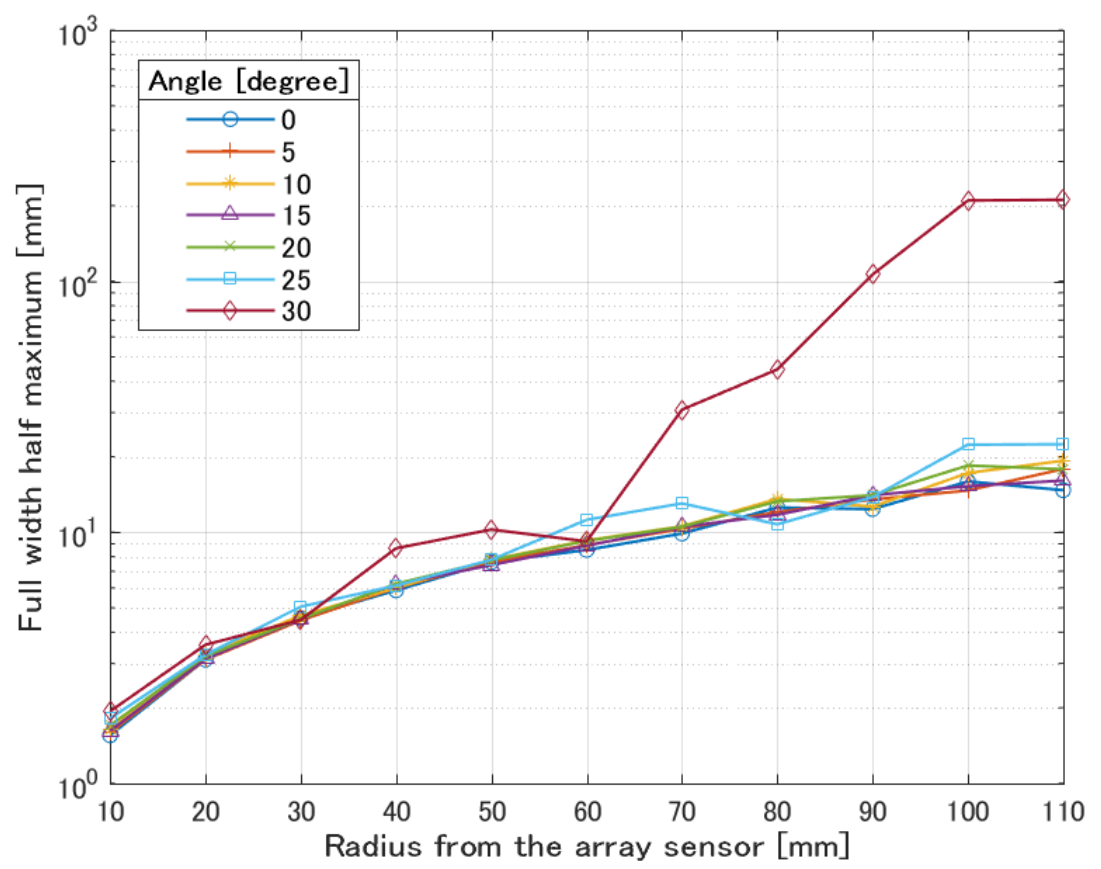

Figure 9: Relationship between the HWHM, the measuring distance and the angle.

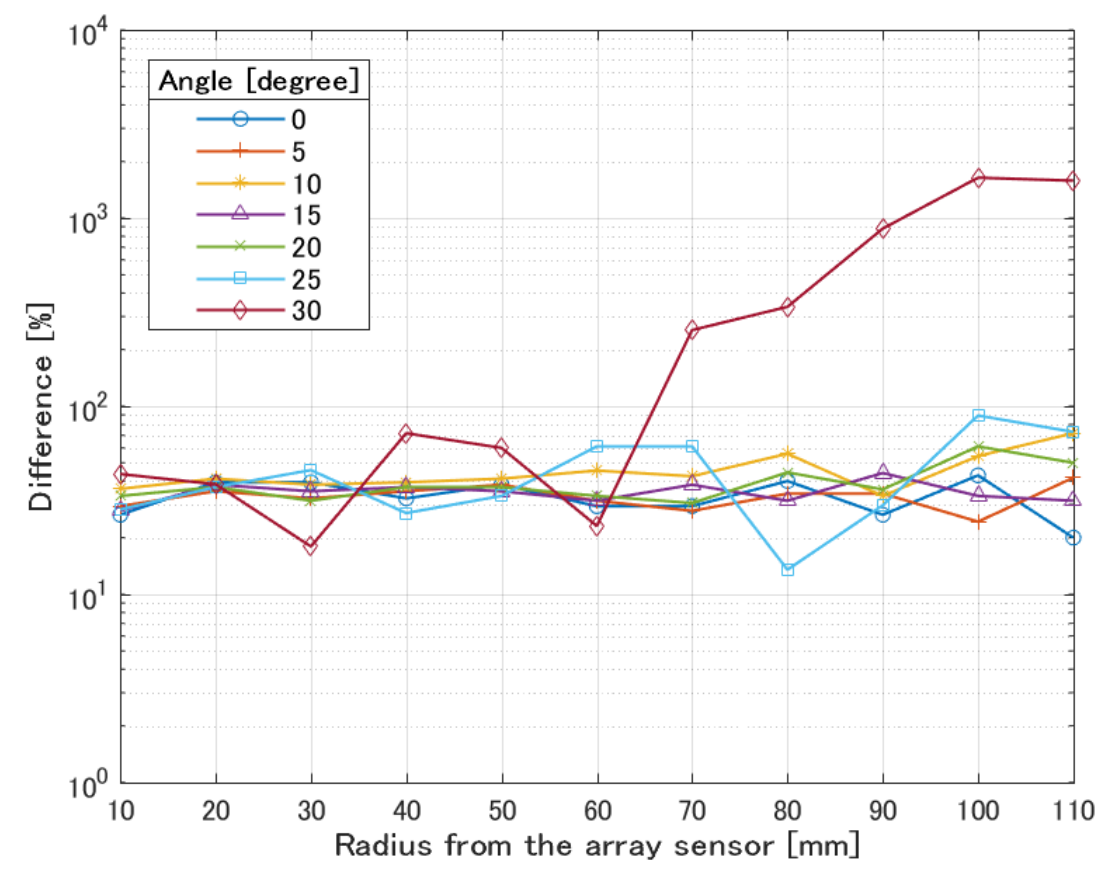

Figure 10: Difference between the HWHM of the DW and the classical TFM. 


\section{DEVELOPMENT}

A measurement system was developed to carry out echo-PIV using the DW-TFM. The measurement device (linear array sensor, pulser/receiver and control PC) used for the data acquisition was the same as the one used in Section 2.3. In the developed system, the DW was repeated at a certain interval over a number of repetitions $N_{\text {rep }}$. In the meanwhile, the acquired data was temporarily stored in the memory of the pulser/receiver. After completing all the pulse repetition, the stored data were transferred and post-processed using eqn (2) to obtain the echo images in the control PC. Those echo images were processed as shown in the flow chart in Fig. 11. The route on the left side is the PIV processing for obtaining the VFM. In this part, the FFT-based cross-correlation method [11] was used as the PIV algorithm. The right route is the auto-correlation part to determine surface of stationary objects in the imaging area. The auto-correlation estimates displacement of the echo intensity, $I(x, z)$ between two subsequent images in time and therefore it is expected to show stationary part in echo images as a support for the VFM. Note that the equation of the auto-correlation is expressed as:

$$
R_{n}(x, z)=\left|I(x, z)_{n}^{*} \cdot I(x, z)_{n+1}\right|,
$$

where subscript of $n$ is the index of the number of repetitions, the symbol of * is the complex conjugate and $R$ is the auto-correlation function.

The image-processing on both the routes was repeated over $N_{\text {rep }}-1$ image pairs and the average of $V$ and $R$ were obtained in the end of the process flow. The whole process in the flow chart was carried out in the Matlab (R2017, MathWorks).

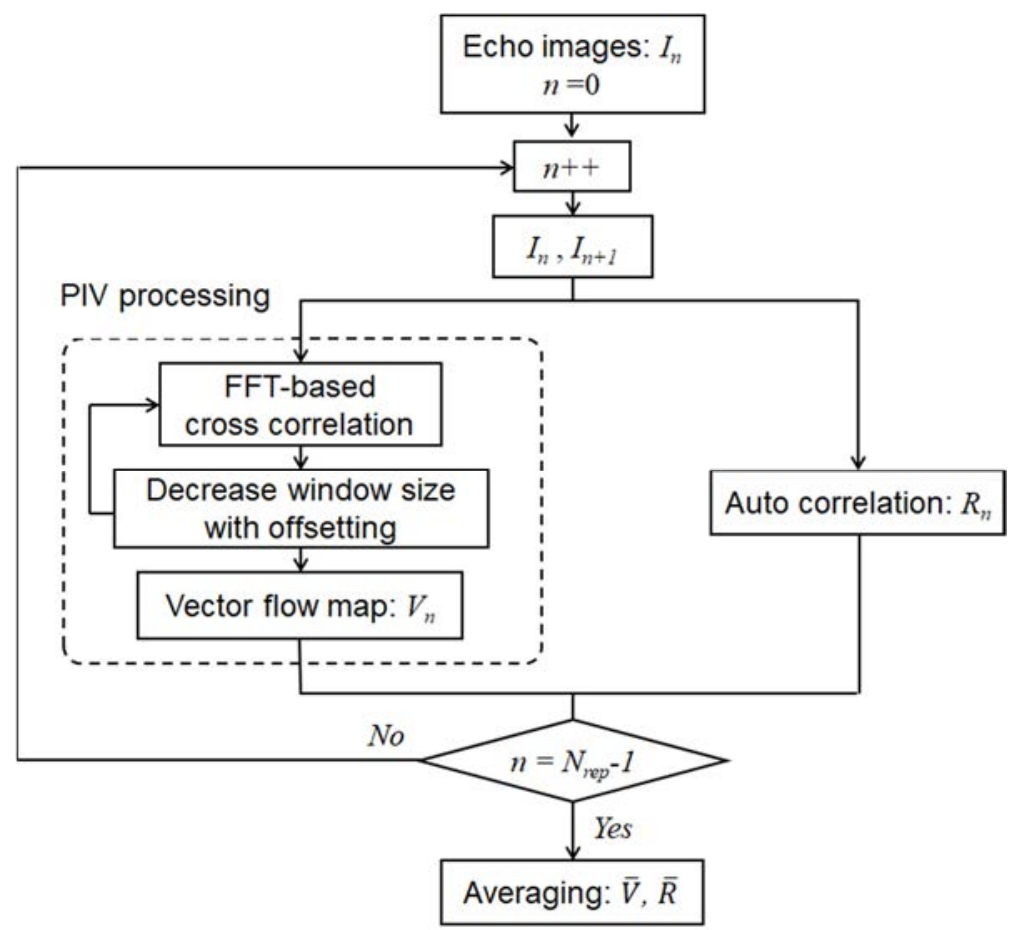

Figure 11: Flow chart of imaging process. 


\section{EXPERIMENT}

In order to verify the applicability of the developed echo-PIV system, an experimental apparatus was created. Fig. 12(a) shows the experimental apparatus and the developed system. The experimental apparatus consists of a water tank $(1280 \times 450 \times 450 \mathrm{~mm})$, inverter, magnetic pump, flowmeter and a test object: a mock fuel debris. During the operation of the apparatus, tracer particles (polyurethane particle: $\varphi 80 \mu \mathrm{m}$ ) were dispersed in the water. The water was circulated using the pump, the rotation speed of which was controlled by the invertor. In this experiment, the outlet of the water tank $(\varphi 19 \mathrm{~mm})$, which was located $290 \mathrm{~mm}$ away from the right wall of the water tank, was regarded as a leakage point and the flow rate was monitored by the flowmeter. Fig. 12(b) shows the detail of the measurement area. The array sensor was located $80 \mathrm{~mm}$ right of the leakage and $110 \mathrm{~mm}$ above the bottom of the water tank under the angle of $35^{\circ}$.

The condition of the data acquisition was the same as the ones in Section 2.3. The measurement area was $x$ from -100 to $100 \mathrm{~mm}$ and $z$ from 0 to $150 \mathrm{~mm}$ (with a $0.5 \mathrm{~mm}$ steps) using the coordinate system shown in Fig. 4. The interval between repetitions was $20 \mathrm{~ms}$ and the number of repetitions was 64 . The water temperature was $17.1^{\circ} \mathrm{C}$ and the flow rate was $0.85 \mathrm{l} / \mathrm{min}$ (the mean velocity inside the outlet was $50 \mathrm{~mm} / \mathrm{s}$ ).

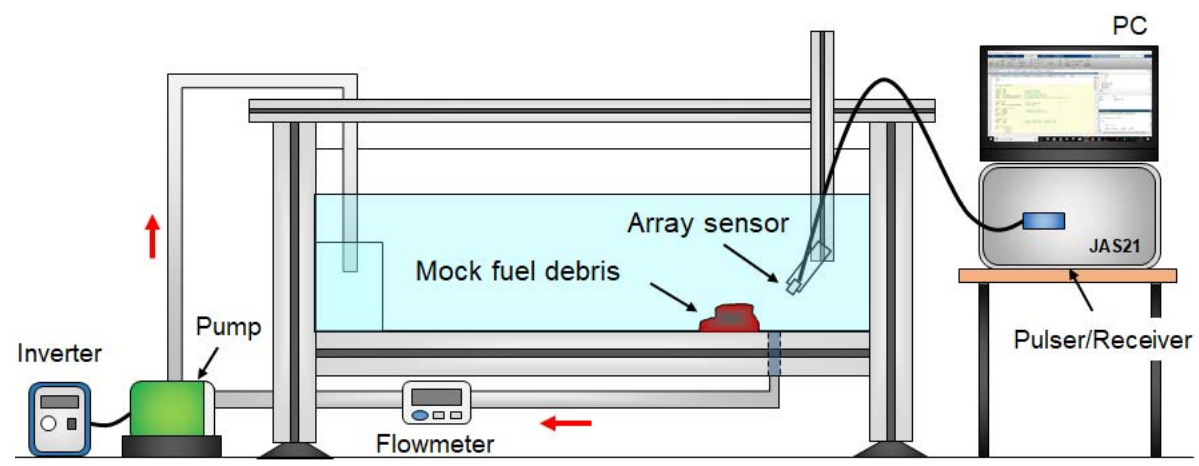

(a)

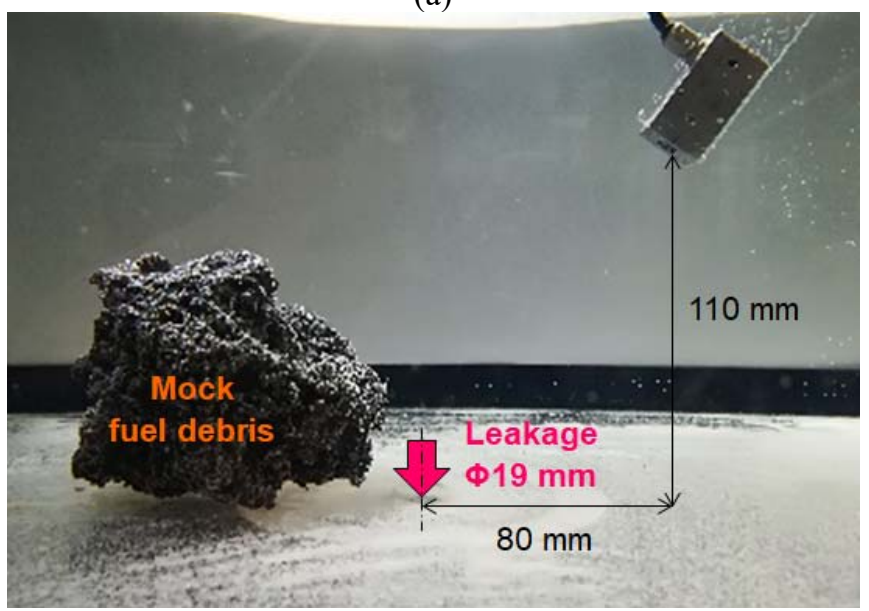

(b)

Figure 12: Overview of the experiment. (a) Schematic of the experimental apparatus; (b) Detail of the measurement area. 


\section{RESULTS AND DISCUSSION}

Fig. 13 shows the result of the experiment as a combination of the $\bar{V}$ and $\bar{R}$ defined in Fig. 11. $\bar{V}$ is color-mapped using its scalar value, and $\bar{R}$ is normalized with its maximum value and plotted as a gray scale image using the experimental geometry. Additionally, the results are displayed only inside the $\pm 30^{\circ}$ area from the center of the array sensor. This result shows a unitary flow direction and a rough stationary object and it allows to assume the location of the leakage at around 70-90 $\mathrm{mm}$ in distance and $100-110 \mathrm{~mm}$ in depth. The actual location of the leakage was between $71-89 \mathrm{~mm}$ in distance and $110 \mathrm{~mm}$ in depth as shown in Fig. 12(b), so the position assumed from the experimental data has a good agreement with that actual location. In the meanwhile, the roughly determined shape of the mock fuel debris can help identifying the location of the leakage as expected. However, the shape was not detected clearly because of the low azimuthal resolution, which deteriorated with the distance from the array sensor. Also, this makes it difficult for the PIV processing to estimate the velocity vector near objects because the echo signal from objects is much stronger than that of tracers and dominates the local echo intensities near object. This phenomenon appears near the leakage and around the mock fuel debris in Fig. 12(b). To deal with the problem, it is considered to change the sensor, since the used array sensor has a limited azimuthal resolution due to its small aperture width and the DW-TFM can be used with other array sensors.

The total scanning time for the data acquisition was $1.3 \mathrm{sec}$ in the experiment. The time does not change for other array sensors and can be shortened by optimizing the transmit interval in accordance with the target velocity. The total data processing time can be shortened by applying ideas from [12] and [13]. Consequently, the technique can potentially achieve a real-time or nearly real-time measurement.

As summary of the experiment, applicability of echo-PIV using the DW-TFM was confirmed.

\section{CONCLUSION}

The echo-PIV was developed to be an inspection method for identifying the location of leakages inside the PCVs at $1 \mathrm{~F}$. In this paper, the DW-TFM was proposed as an imaging
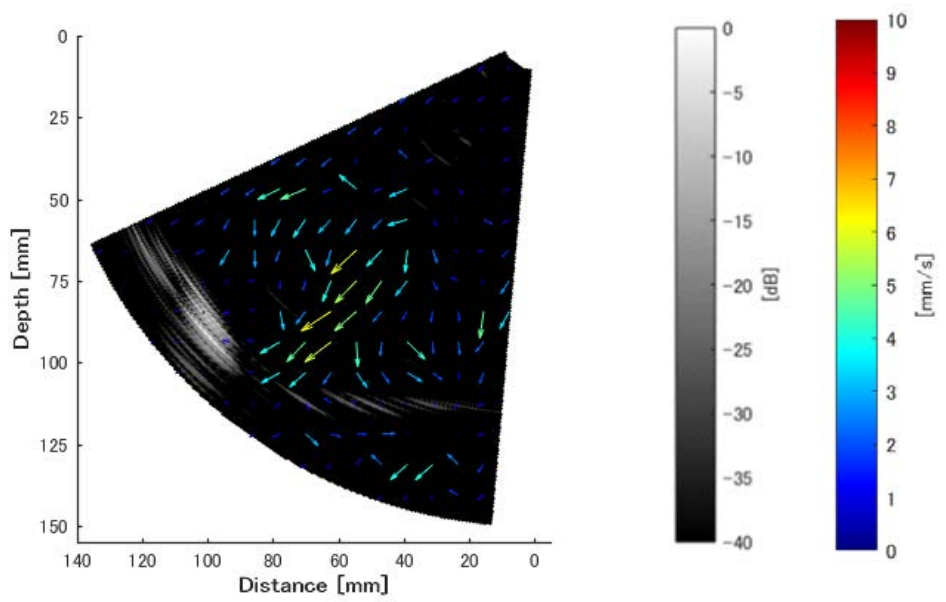

Figure 13: Experimental result. 
method to improve the performance of the echo-PIV for the inspection. After investigating the imaging performance of the DW-TFM, a measurement system was developed to carry out the echo-PIV using the DW-TFM. To verify the applicability of the developed system, an experiment simulating the situation inside PCVs was conducted. As a result, the location of the leakage from the measurement has a good agreement with the actual location. Therefore, the developed system has a potential to fulfill all the requirements for the inspection technique, which could be used inside the PCVs of the 1F.

\section{ACKNOWLEDGEMENT}

The authors express their sincere gratitude to Japan Probe Co. Ltd., for providing the pulser/receiver for this research.

\section{REFERENCES}

[1] Tokyo Electric Power Company Holdings (TEPCO) Inc., Progress report on the decommissioning of Fukushima Dai-ichi nuclear power plant. www.meti.go.jp/ earthquake/nuclear/decommissioning/committee/fukushimahyougikai/2017/pdf/ 0929 01e.pdf. Accessed on: 10 Apr. 2018.

[2] Beller, L.S. \& Brown, H.L., Design and operation of the core topography data acquisition system for TMI-2, Three Mile Island Reports, GEND-INF-012, 1984.

[3] Hou Su-xia, L.J., Bin, H., Ru-song, L. \& Tao, S., The treatment of radioactive wastewater by ultrasonic standing wave method. Journal of Hazardous Materials, pp. 41-45, 2014.

[4] Batsaikhan, M., Hamudani, A. \& Kikura, H., Velocity measurement on two-phase air bubble column flow using array ultrasonic velocity profiler. International Journal of Computational Methods and Experimental Measurements, 6(1), pp. 86-97, 2018.

[5] Takeda, Y., Development of an ultrasound velocity profile monitor. Nuclear Engineering and Design, 126, pp. 277-284, 1991.

[6] Kim, H.B., Herzberg, J.R. \& Shandas, R., Development and validation of echo PIV. Experiments in Fluids, 36, pp. 455-462, 2004.

[7] Holmes, C., Drinkwater, B.W. \& Wilcox, P.D., Post-processing of the full matrix of ultrasonic transmit-receive array data for non-destructive evaluation. NDT \& E International, 38, pp. 701-711, 2005.

[8] Hasegawa, H. \& Kanai, H., High-frame rate echocardiography using diverging transmit beams and parallel receive beamforming. J. Med. Ultrason., 38, pp. 129-140, 2011.

[9] Ahmad, R., Kundu, T. \& Placko, D., Modelling of phased array transducers. The Journal of Acoustic Society of America, 117, pp. 1762-1776, 2005.

[10] Ihara, T., Kawachi, T., Fukumoto, T., Tsuzuki, N., Kikura, H. \& Kimoto, K., Fundamental study of ultrasonic measurement for leakage flow mapping and debris inspection. Proceedings of the 26th International Symposium on Transport Phenomena, pp. 1-4, 2015.

[11] Stamhuis, E.J., Basics and principles of particle image velocimetry (PIV) for mapping biogenic and biologically relevant flows. Aquatic Ecology, pp. 463-479, 2006.

[12] Rougeron, G., Lambert, J., Iakovleva, E., Lacassagne, L. \& Dominguez, N., Implementation of a GPU accelerated total focusing reconstruction method within CIVA software. AIP Conference Proceedings, pp. 1983-1990, 2014.

[13] Champagnat, F., Plyer, A., Le Besnerais, G., Leclaire, B., Davoust, S. \& Le Sant, Y., Fast and accurate PIV computation using highly parallel iterative correlation maximization. Exp. Fluid., pp. 1169-1182, 2011. 